\title{
Development of Mechanical Engineering Experiment Platform for Virtual Instrument
}

\author{
Man Li \\ Xi’an International University, Xi’an, Shaanxi, 710077
}

Keywords: Mechanical Engineering, Virtual Instrument, Experiment Platform Construction

\begin{abstract}
In this paper, the mechanical engineering innovation laboratory as the research background, in close connection with the mechanical engineering test technology this professional course experimental teaching requirements, Labview is virtual instrument software as a platform, based on mechanical engineering test theory and sensor technology, developed a mechanical engineering Test bench, its functions include: software can be achieved through the data collection, analysis and processing and experimental results show; also can carry out a series of mechanical engineering parameters of the test.
\end{abstract}

\section{Introduction}

Testing is an important and effective means of testing the function of the test object, whether the performance meets the requirements and promptly identifies the problem. In general, in the product development, production and use of the maintenance process are inseparable from the test technology and test equipment, test technology is an important means of product development process, product manufacturing process is the quality assurance tool, but also the product Key elements of maintenance assurance system. In particular, today's automatic control technology has been more and more use of test technology, test equipment has become an important part of the control system. With the development of new technologies in the world, the application of information technology and information equipment has become a key way to improve the equipment capacity, and has been widely used in the fields of aerospace, aviation, ship, vehicle and so on. Whether it is civil or military field of land and sea and air equipment, communications systems, automated production devices, etc., the electronic equipment is also constantly increasing, in order to ensure the normal work of equipment, these advanced and complex electronic equipment testing and failure Diagnosis is particularly important. Specific to mechanical engineering, such as a mechanical or mechanical, from design, manufacture, operation, maintenance to the final scrapped, are inseparable from mechanical testing and measurement. Modern machinery and equipment dynamic analysis, process detection and control, product quality inspection, equipment, modern management, condition monitoring and fault diagnosis, have to rely on mechanical testing. Mechanical testing is the technical basis for the realization of these processes, as well as technical means for scientific exploration, scientific discovery and technological inventions. Therefore, the testing technology is an important base technology for the development of machinery industry.

\section{Construction of Mechanical Engineering Test Bench}

The so-called virtual instrument refers to the general computer as the core of the hardware platform, the user's own design and definition of a virtual operation panel, test function by the test software to achieve a computer instrument system. Virtual instruments break through the traditional experimental instruments in the form of hardware as the core. In fact, the experiment is in the operation of the computer with the relevant test software, like the operation of virtual test equipment, virtual instrument hence the name. "Software is the instrument", the most essential to characterize the virtual instrument. It is more common than traditional instruments, virtual instruments can adapt to the rapid development of contemporary science and technology, and in accordance with the latest science and technology to achieve real-time update requirements, to 
promote the traditional instrument in the digital, modular, virtual, network direction development of. Many types of test equipment have different functions. But the various test equipment, its composition can be summarized as data collection and control, data analysis and processing, the results of the output and display three functional modules, and are in the form of hardware, so the development and maintenance of high cost, technology Update cycle length Even later the emergence of digital instruments, intelligent instruments, so that the accuracy of traditional instruments to improve, enhance the function, but has not changed the traditional instrument that the use of independent, manual operation, a single task mode. To this end, the bus-type instruments and systems came into being, people developed a variety of communication interfaces, used to connect multiple intelligent instruments together to form a more functional, to adapt to a wider test system, but this bus instrument There are still many repetitive parts or functional units.

The fundamental reason for determining the characteristics of virtual instruments that are not possible with traditional instruments is that "the key to virtual instruments is software." Virtual instruments compared with traditional instruments, have the following characteristics:

There is only one panel of traditional instruments, which are arranged with a wide variety of display and operation elements, which are prone to many identification and operation errors. Virtual instruments are different, it can be in several sub-panel operation to achieve more complex functions. At the same time, the types and forms of display elements and operating elements on the virtual instrument panel are not limited by the "standard parts" and "processing processes", which are implemented by programming and the designer can design according to the user's cognitive requirements and operational requirements Instrument panel.

After the general hardware platform is determined, the software is used to replace the hardware in the traditional instrument to complete the function of the instrument.

The function of the instrument is defined by the software as needed by the user, not by the manufacturer.

Instrument performance improvements and functional expansion only need to update the relevant software design, do not need to buy a new instrument.

Development cycle is much shorter than traditional instruments.

Virtual instrument is open, flexible, can be synchronized with the development of the computer, and the network and other peripheral devices.

It can be different instruments functionally integrated, with a dedicated hardware board with a virtual instrument can achieve the function of multiple instruments. The analysis, display, storage, printing and management of the signal to the computer to deal with, make full use of computer technology to complete the data transmission and exchange performance, making the system become flexible and simple. The software acts as a former hardware. Reduce the number of possible drift with time, need to regularly calibrate the separation of analog hardware, the use of standardized bus, the system measurement accuracy, speed are greatly improved.

\section{Design of Test Bench for Mechanical Engineering Test of Virtual Instrument}

The goal of the mechanical engineering test bench is to develop students' innovative ability and practical ability to train students in the application of test system for the detection of mechanical quantities, to understand and master such as modern sensor technology, data acquisition and processing technology, virtual instrument technology And other aspects of knowledge and strengthen the application.

On the basis of satisfying the experimental teaching and scientific research, this experimental platform can not only alleviate the shortage of experiment, but also benefit the flexibility and diversification of the experiment, in order to make up the deficiency of the traditional experimental instrument and replace the traditional instrument with the virtual instrument. The experimental content can be achieved not only in close contact with teaching, but also with the combination of engineering practice, has some practical significance, students no longer stay in the verification experiment, but can be exposed to engineering practice is closely related to innovative Experiment, not only to solve the students for mechanical engineering testing technology this course problems, 
but also through the experimental development of students' innovative thinking, improve the fun of the experiment. In order to make the students fully understand and master the structure and application of the mechanical engineering test system, the whole experiment system is divided into two sections: teaching assistant courseware and engineering innovation experiment. In particular, several typical test experiments were set up to increase the proportion of design experiment.. Help students to strengthen the understanding and understanding of the structure and design of the test system. At the same time fully exercise the ability of students to use knowledge and practical ability to adapt to the development and requirements of the new era of education.

\section{Experimental Platform Software System Design}

The software design of the experimental platform is based on the Labview software. The function of the experimental platform is combined with the general process of the test system, which can realize the functions of data acquisition, data analysis and processing, and data printing. Data acquisition (DAQ), is to measure the various parameters of the object (such as chemical quantity, physical quantity, biomass, etc.) through a variety of sensors to collect and do the appropriate conversion, and then through the signal conditioning, sampling, quantization, coding, Transmission and other steps to the controller for data recording or processing process. The controller is typically borne by the computer. The computer is the core of the entire data collection process, which controls the system and processes the data after the data is collected. The acquisition process is the first through the sensor will be measured by the physical quantity into electrical signals, and through the signal conditioning module for pre-processing; and then through the preprocessing of the analog signal through the data acquisition card can be converted to computer can identify the digital signal, in addition to Zoom, sample hold and multiplexing.

The data acquisition module is designed as the core of the entire data acquisition system, which collects any data that requires real-time analysis into the computer, which directly affects the results of subsequent data analysis and processing, as well as other functions of the system achieve. So the module can be normal and accurate operation is directly related to the entire system can work properly. The data acquisition module sets the parameters of the related parameters (sampling frequency, channel number, sampling maximum and minimum value, input mode configuration, sampling mode, DAQ physical channel selection, etc.) according to the need, and controls the process of data acquisition. The system can support the channel data synchronization acquisition, data acquisition process can be directly dynamic display of data collection, data collection can be completed according to the needs of the collected data in real time processing, data can also be saved to prepare for subsequent calls. In the data acquisition module interface, there are three basic functions such as parameter setting, function operation and waveform display. Before the data acquisition program can be started in the parameter setting area according to the need to set the corresponding parameters to complete the data acquisition card configuration and initialization, the system needs to configure the parameters are: channel selection, sampling the maximum and minimum, sampling Frequency and other parameters. "Channel selection" You can set the number of channels to be collected as needed.

In the virtual instrument, according to the needs of the test of the collected data for a variety of analysis and processing, its purpose is: to truly restore the collected signal; to eliminate the signal interference signal: in the data acquisition, transmission and conversion process, Due to the presence of internal and external disturbances and noise, the interfering signal is mixed in the acquired signal. And therefore need to use a variety of methods to minimize the interference signal mixed with the useful signal to ensure the accuracy of the collected data; analysis of the inherent characteristics of the data: through the collection of data to transform (such as the average or do Fourier, Wavelet transform, etc.), or between the associated data for some interoperability (such as the calculation of correlation function), to be able to express the intrinsic characteristics of the data secondary data. 


\section{Conclusion}

Virtual instrument development in China started relatively late, the research people are relatively small, and the virtual instrument and mechanical engineering test bench combined with a little less in the application. I am in this area due to lack of experience, lack of time, so the experimental bench has a lot of new features and new ideas are not perfect. However, I believe that with the follow-up research and development of the deepening of the virtual instrument in the field of mechanical engineering applications will be more and more attention, many of the problems that are not resolved in the future will certainly be resolved. I have been close to a year of research, in the development of the experimental platform has made some achievements. But the design of the test bench there are still some shortcomings.

\section{References}

[1] Xing Kanglin, Shajie. Construction and application of precision grinding monitoring and feedback system for medium and small holes [J]. Combined machine tools and automated machining technology, 2013 (08)

[2] Sha Jie, Zhao Haizhou. Application of AE sensor in grinding of inner and outer wells [J]. Mechanical Engineer, 2013 (08)

[3] Deng Shuping, Xu Guowei. Design and research of adaptive filter based on Labview [J]. Microcomputer Applications, 2011 (03)

[4] Kong Yan, Liu Zhengran. Laboratory construction and management of colleges and universities [J]. Chinese community physician (medical professional) .208 (21)

[5] Jiang Jingying, Xu Kexin, Li Hao, Li Nan. Design of experimental system based on Labview data acquisition and analysis [J]. Laboratory Science, 2009 (06)

[6] Ma Yinping, Xuan Liangliang, Peng Ru. Based on Labview data acquisition system analysis and design [J]. Electronic Components Applications, 2009 (10) 